\title{
Efficient Electromagnetic Analysis of Line-Fed Aperture Antennas in Thick Conducting Screens
}

\author{
Ivica Stevanović, Student Member, IEEE, and Juan R. Mosig, Fellow, IEEE
}

\begin{abstract}
This paper presents a numerical and experimental verification of an approximate but efficient integral equation technique for the scattering by apertures in conducting planes with finite thicknesses. The approach is based on a perturbation method and modified Green's functions that take into account the finite metallization thickness. The computational effort and time needed for solving the problem are the same as in zero-thickness case. When compared to full-wave cavity treatment of thick apertures, the method is (depending on the number of unknowns) at least an order of magnitude faster. The method can be applied even to apertures of arbitrary shapes where computing the cavity's Green's functions is a difficult task. The results of simulations using the new approach show good agreement when compared to both results from full-wave cavity approach and measurements.
\end{abstract}

Index Terms-Green's functions, integral equations (IE), multilayered structures, scattering by thick apertures, slot antennas, stratified media theory.

\section{INTRODUCTION}

A LARGE number of radiating structures including apertures is traditionally analyzed considering the aperture made in a zero-thickness screen. These structures include slot-fed patch antennas, cavity backed antennas with feeding irises, waveguide slot antennas and the simple slot antenna fed by a printed line. However, the use of new technologies (for instance, self-standing bulk ground planes instead of printed ones) and the drive for higher frequencies are calling for the inclusion of finite thickness effects in the currently existing models.

Harrington and Mautz [1] were the first who addressed this problem employing a transmission line model. Other researchers have recently followed this approach [2], [3] to compute aperture coupled microstrip antennas. A similar approach, based on reciprocity has been reported in [4]. Another way to tackle the problem is to treat the aperture volume as a cavity or a waveguide. In [5] and [6] the integral equation (IE) formulation is combined with finite-element method (FEM) for the cavity. These methods are very versatile, and able to model arbitrary cross sections filled with inhomogeneous dielectrics. However, both require a separate FEM solution to be integrated into the IE framework. The mixed potential integral equation (MPIE) formulation for the outer regions combined with an IE field formulation for the cavity (aperture), using a

\footnotetext{
Manuscript received June 12, 2003; revised November 19, 2003. This work was supported in part by ESA-ESTEC under Contract 12996/98/NL/DS.

The authors are with the Laboratory of Electromagnetics and Acoustics, Ecole Polytechnique Fédérale de Lausanne, CH-1015 Lausanne, Switzerland (e-mail: ivica.stevanovic@epfl.ch; juan.mosig@epfl.ch).

Digital Object Identifier 10.1109/TAP.2004.835268
}

modal expansion of the Green's function, is outlined in [7]. This method has the advantage of a consistent IE approach, which produces well-conditioned matrices and leads to highly accurate results. Also several layers of dielectrics even with embedded metallizations can be treated within the apertures. A drawback of the method is the difficulty in computing the required modal functions, since computations in cross-sections other than rectangular and circular ones become a difficult task. Moreover, for any aperture geometry, the summation of the modal series calls for specially tailored acceleration schemes [8], [9]. Recently, another IE formulation, a full wave mixed spectral/spatial method of moments (MoM), has been reported [10]. In this approach, the thick slot is modeled by a parallel plate waveguide with vertical electric currents added to account for the metallic walls. The method is capable of modeling apertures of arbitrary thicknesses and shapes filled with arbitrary number of dielectric layers.

The above-mentioned methods can deal with very general aperture problems. But they lack the simplicity and internal coherence of the straightforward IE model (the so-called 2.5 D model) that can be used in the case of zero-thickness apertures. Recently, a new approximate approach for slots on thick screens has been proposed [11]. The slot thickness would appear only as a modification in the Green's functions of the problem, but otherwise the slot could be treated as a two-dimensional object.

This paper fully develops this conjuncture and shows that the aperture model can be, with minor modifications, fully incorporated into the traditional integral equations for slots and patch antennas embedded in stratified media. Indeed, this brings the study of slot thickness effects under the reach of traditional planar IE codes.

After introducing the new complete set of integral equations for a printed-line fed slot antenna, which involves both electric and magnetic currents, this paper explores and unthreads some computational relevant aspects of the model developed in [11] and provides further numerical verifications. Finally, the new complete IE model is validated by comparing its numerical predictions with measurements for a set of slot antennas with different shapes and thicknesses.

\section{Formulation OF INTEGRAL EQUATIONS}

Consider a structure composed of a planar printed line or patch and an aperture of finite thickness $t=z_{2}-z_{1}$ embedded into stratified dielectric layers as shown in Fig. 1. Using the equivalence principle, the interfaces of the aperture are shortcircuited, i.e., continuous perfect electric screens are introduced at $z=z_{1}$ and $z=z_{2}$ and the continuity of the tangential components of the electric fields on aperture interfaces is ensured 


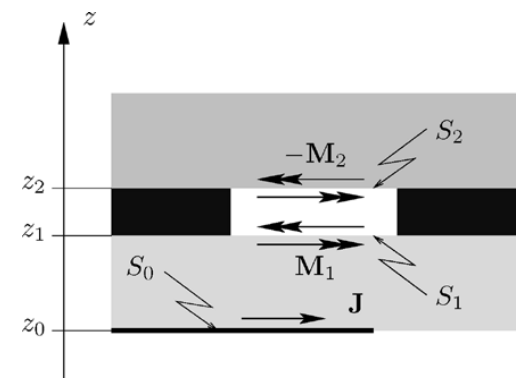

Fig. 1. Aperture in a thick conducting shield embedded in a layered structure.

by introducing the equivalent surface magnetic currents $\mathbf{M}_{1,2}$ [12]. This way, the original problem can be decoupled into three equivalent ones: lower region $\left(z<z_{1}\right)$, closed aperture or cavity region $\left(z_{1} \leq z<z_{2}\right)$, and upper region $\left(z \geq z_{2}\right)$. Assuming an incident electromagnetic field $\left(\mathbf{E}^{i}, \mathbf{H}^{i}\right)$ present in the lower region $z<z_{1}$, the boundary conditions at the patch and the lower and the upper interface of the aperture become

$$
\begin{aligned}
\hat{z} \times \mathbf{E}\left(\mathbf{r}_{0}\right) & =-\hat{z} \times \mathbf{E}^{\mathrm{exc}}, \quad \mathbf{r}_{0} \in S_{0} \\
\hat{z} \times\left[\mathbf{H}^{+}\left(\mathbf{r}_{1}\right)-\mathbf{H}^{-}\left(\mathbf{r}_{1}\right)\right] & =\hat{z} \times \mathbf{H}^{\mathrm{exc}}, \quad \mathbf{r}_{1} \in S_{1} \\
\hat{z} \times\left[\mathbf{H}^{+}\left(\mathbf{r}_{2}\right)-\mathbf{H}^{-}\left(\mathbf{r}_{2}\right)\right] & =0, \quad \mathbf{r}_{2} \in S_{2}
\end{aligned}
$$

where $\mathbf{r}_{i}, i=0,1,2$ is the position vector with respect to the global coordinate system, $\mathbf{H}^{ \pm}$and $\mathbf{E}$ are the scattered fields produced by magnetic $\left(\mathbf{M}_{1,2}\right)$ and electric $(\mathbf{J})$ currents, and the superscripts (+) and ( - ) refer to, respectively, the regions above and below the corresponding $z$-coordinate. $\mathbf{H}^{\text {exc }}$ is the sum of the incident magnetic field $\mathbf{H}^{i}\left(\mathbf{r}_{1}\right)$ and its image (so-called short-circuit magnetic field [13]) and $\mathbf{E}^{\mathrm{exc}}=\mathbf{E}^{i}\left(\mathbf{r}_{0}\right)$. Following the procedure outlined in [11], all the scattered fields are expressed as convolutions between the corresponding sources and the pertinent stratified media Green's functions. Also, we use the strategy suggested in [11] and introduce another couple of magnetic unknowns, the half-sum $\mathbf{M}_{\Sigma}=\left(\mathbf{M}_{1}+\mathbf{M}_{2}\right) / 2$ and the half-difference $\mathbf{M}_{\Delta}=\left(\mathbf{M}_{1}-\mathbf{M}_{2}\right) / 2$. Moreover, in the closed aperture region, taking into account the symmetry and reciprocity [14], we only need two different Green's functions of the magnetic-magnetic (HM) type, namely, $\stackrel{\mathrm{G}}{\mathrm{HM}}_{\mathrm{HM}}^{(c)}$ (the cavity Green's function with source and observer at the same horizontal interface) and $\overleftrightarrow{\mathrm{G}}_{\mathrm{HM}}^{(c) \times}$ (the cavity Green's function with source and observer at different horizontal interfaces). Then, according to [11], we define the sum and the difference cavity Green's functions as

$$
\begin{aligned}
& \overleftrightarrow{\mathbf{G}}_{\mathrm{HM}}^{(c) \Sigma}=\overleftrightarrow{\mathbf{G}}_{\mathrm{HM}}^{(c)=}+\overleftrightarrow{\mathbf{G}}_{\mathrm{HM}}^{(c) \times} \\
& \overleftrightarrow{\mathbf{G}}_{\mathrm{HM}}^{(c) \Delta}=\overleftrightarrow{\mathbf{G}}_{\mathrm{HM}}^{(c)=}-\overleftrightarrow{\mathbf{G}}_{\mathrm{HM}}^{(c) \times}
\end{aligned}
$$

Introducing all these definitions in the boundary condition (1), we obtain, after some straightforward algebraic manipulations, the set of coupled integral equations (Fig. 1) shown in (3a)-(3c) at the bottom of the page, where $\otimes$ is a shorthand notation for the convolution integral, $\mathbf{E}_{t}^{\text {exc }}=\hat{z} \times \mathbf{E}^{\text {exc }}$, and $\mathbf{H}_{t}^{\text {exc }}=\hat{z} \times \mathbf{H}^{\text {exc }}$. $\overleftrightarrow{\mathbf{G}}_{\mathrm{EJ}}$ is the electric field Green's function with both observer and electric source situated at $z=z_{0}, \overleftrightarrow{\mathrm{G}}_{\mathrm{EM}}$ is the electric field Green's function with observer at $z=z_{0}$ and magnetic source at $z=z_{1}$, and $\overleftrightarrow{\mathbf{G}}_{\mathrm{HJ}}$ is the magnetic field Green's function with observer at $z=z_{1}$ and electric source situated at $z=z_{0}$. These three Green's functions should satisfy the boundary conditions for fields on the perfect electric screen situated in the plane $z=$ $z_{1}$. In addition to the cavity Green's function, two more Green's functions of HM type are needed $\overleftrightarrow{\mathrm{G}}_{\mathrm{HM}}^{(1)}$-Green's function of the stratified lower half-space with both source and observer at $z=z_{1}$ and the boundary conditions for magnetic field satisfied on the perfect electric screen at $z=z_{1}$; and $\overleftrightarrow{\mathrm{G}}_{\mathrm{HM}}^{(2)}$-Green's function of the stratified upper half-space with both source and observer at $z=z_{2}$ and the boundary conditions for magnetic field satisfied on the perfect electric screen at $z=z_{2}$.

In apertures of practical thicknesses, there exists a rather strong correlation between $\mathbf{M}_{1}$ and $\mathbf{M}_{2}$, and therefore it is reasonable to assume that the difference of the two currents is zero $\mathbf{M}_{\Delta} \approx 0$. This approximation, which is exact in the limiting zero-thickness case, transforms the system (3) into

$$
\begin{array}{r}
\overleftrightarrow{\mathbf{G}}_{\mathrm{EJ}} \otimes \mathbf{J}+\overleftrightarrow{\mathbf{G}}_{\mathrm{EM}} \otimes \mathbf{M}_{\Sigma}=-\mathbf{E}_{t}^{\text {exc }} \\
\left(\overleftrightarrow{\mathbf{G}}_{\mathrm{HM}}^{(1)}+\overleftrightarrow{\mathbf{G}}_{\mathrm{HM}}^{(2)}+2 \overleftrightarrow{\mathbf{G}}_{\mathrm{HM}}^{(c) \Delta}\right) \otimes \mathbf{M}_{\Sigma}-\overleftrightarrow{\mathbf{G}}_{\mathrm{HJ}} \otimes \mathbf{J}=-\mathbf{H}_{t}^{\text {exc }} .
\end{array}
$$

The set of equations (4) clearly shows that the strategy introduced in [11] can be generalized to more complex structures involving both electric and magnetic currents. Indeed, (4) is identical to the zero-thickness case save for the use of $\mathbf{M}_{\Sigma}$ instead of a unique current $\mathbf{M}$ and the correcting term $2 \overleftrightarrow{\mathbf{G}}_{\mathrm{HM}}^{(c) \Delta}$. In principle, the remaining equation (3b) could be used afterwards to find a nonzero estimation of $\mathbf{M}_{\Delta}$, and eventually start an iterative process.

\section{Calculation of the CaVity Green's Functions}

By introducing the electric and magnetic surface charge densities $\rho=-(1 / j \omega) \nabla \cdot \mathbf{J}$ and $\rho_{m}=-(1 / j \omega) \nabla \cdot \mathbf{M}$, we can always set up the relation between the field and potential Green's functions [15]

$$
\begin{aligned}
\overleftrightarrow{\mathbf{G}}_{\mathrm{EJ}} \otimes \mathbf{J} & =-j \omega \overleftrightarrow{\mathbf{G}}_{A} \otimes \mathbf{J}-\nabla\left(G_{V} \otimes \rho\right) \\
\stackrel{\mathbf{G}}{\mathrm{HM}} \otimes \mathbf{M} & =-j \omega \overleftrightarrow{\mathbf{G}}_{F} \otimes \mathbf{M}-\nabla\left(G_{W} \otimes \rho_{m}\right)
\end{aligned}
$$

$$
\begin{aligned}
& \overleftrightarrow{\mathbf{G}}_{\mathrm{EJ}} \otimes \mathbf{J}+\overleftrightarrow{\mathbf{G}}_{\mathrm{EM}} \otimes \mathbf{M}_{\Sigma}+\overleftrightarrow{\mathbf{G}}_{\mathrm{EM}} \otimes \mathbf{M}_{\Delta}=-\mathbf{E}_{t}^{\mathrm{exc}} \\
& \left(\overleftrightarrow{\mathbf{G}}_{\mathrm{HM}}^{(1)}+\overleftrightarrow{\mathbf{G}}_{\mathrm{HM}}^{(c) \Delta}\right) \otimes \mathbf{M}_{\Sigma}-\overleftrightarrow{\mathbf{G}}_{\mathrm{HJ}} \otimes \mathbf{J}=\left(\overleftrightarrow{\mathbf{G}}_{\mathrm{HM}}^{(2)}+\overleftrightarrow{\mathbf{G}}_{\mathrm{HM}}^{(c) \Sigma}\right) \otimes \mathbf{M}_{\Delta} \\
& \left(\stackrel{\leftrightarrow}{\mathbf{G}}_{\mathrm{HM}}^{(1)}+\stackrel{\leftrightarrow}{\mathbf{G}}_{\mathrm{HM}}^{(2)}+2 \stackrel{\leftrightarrow}{\mathbf{G}}_{\mathrm{HM}}^{(c) \Delta}\right) \otimes \mathbf{M}_{\Sigma}+\left(\overleftrightarrow{\mathbf{G}}_{\mathrm{HM}}^{(1)}-\overleftrightarrow{\mathbf{G}}_{\mathrm{HM}}^{(2)}\right) \otimes \mathbf{M}_{\Delta}-\overleftrightarrow{\mathbf{G}}_{\mathrm{HJ}} \otimes \mathbf{J}=-\mathbf{H}_{t}^{\text {exc }}
\end{aligned}
$$


where $\overleftrightarrow{\mathbf{G}}_{A}$ and $G_{V}$, and $\overleftrightarrow{\mathbf{G}}_{F}$ and $G_{W}$ are the dyadic and scalar potential Green's function for the electric and magnetic sources, respectively. With the MPIE formulation, the correction term will consist of the self- and mutual interactions of the potential cavity Green's functions

$$
\begin{aligned}
\overleftrightarrow{\mathbf{G}}_{F}^{(c) \Delta} & =\overleftrightarrow{\mathbf{G}}_{F}^{(c)}=-\overleftrightarrow{\mathbf{G}}_{F}^{(c) \times} \\
G_{W}^{(c) \Delta} & =G_{W}^{(c)=}-G_{W}^{(c) \times} .
\end{aligned}
$$

The cavity Green's functions can be expanded in terms of the eigenfunctions that depend on the shape of the cavity cross-section. However, if the lateral dimensions of the cavity are big enough compared to its length (large aperture), the guiding properties of the corresponding shallow cavity will be weak. And with the problem discretized into a number of subsectional basis functions, the appropriate currents in the center of the aperture will be only slightly affected by the cavity borders. Therefore, the problem can be treated as a parallel plate problem. The approximation will not be very accurate for the current coefficients belonging to the basis functions near the cavity borders. However, the form of the cavity is taken into account by the lateral distribution of the basis functions. Approximating the rigorous cavity formulation we avoid at the same time the summation of slowly convergent modal series [8], [9], and, maybe even more advantageous, the evaluation of the modal eigenfunctions for cavities with cross-sections of arbitrary shapes. It should be noted, however, that this approximation will remain valid for all shapes of the aperture as long as the aperture thickness is sufficiently small compared to its minimal lateral dimension.

For a parallel plate problem, the equivalent transmission line network given in Fig. 2 is valid. The excitation with a voltage generator corresponds to a magnetic surface current on the interface and yields the two currents

$$
\begin{aligned}
& I_{1}^{\mathrm{TM} / \mathrm{TE}}=\frac{1}{2 \pi} \frac{1}{j Z_{c}^{\mathrm{TM} / \mathrm{TE}} \tan \beta t} \\
& I_{2}^{\mathrm{TM} / \mathrm{TE}}=\frac{1}{2 \pi} \frac{1}{j Z_{c}^{\mathrm{TM} / \mathrm{TE}} \sin \beta t}
\end{aligned}
$$

where $Z_{c}^{\mathrm{TM} / \mathrm{TE}}$ is the characteristic impedance of the respective mode, namely, $\beta /(\omega \varepsilon)$ for the TM mode and $\omega \mu / \beta$ for the TE mode, and $t=z_{2}-z_{1}$ is the thickness of the considered aperture.

The correction terms of the potential Green's functions are then obtained applying the zero-order inverse Sommerfeld transformation [16]

$$
\begin{aligned}
G_{F}^{(c) \Delta x x} & =G_{F}^{(c) \Delta y y}=\mathcal{S}_{0}\left[\frac{1}{j \omega}\left(I_{1}^{\mathrm{TM}}-I_{2}^{\mathrm{TM}}\right)\right] \\
G_{W}^{(c) \Delta} & =\mathcal{S}_{0}\left[\frac{j \omega}{k_{\rho}^{2}}\left(I_{1}^{\mathrm{TE}}-I_{2}^{\mathrm{TE}}-\left(I_{1}^{\mathrm{TM}}-I_{2}^{\mathrm{TM}}\right)\right)\right] .
\end{aligned}
$$

It should be noted that both currents $I_{1,2}^{\mathrm{TM} / \mathrm{TE}}$ go to infinity when the thickness of the aperture $t$ approaches zero. However, their difference $I_{1}^{\mathrm{TM} / \mathrm{TE}}-I_{2}^{\mathrm{TM} / \mathrm{TE}}$, proportional to $\tan (\beta t / 2)$, con-

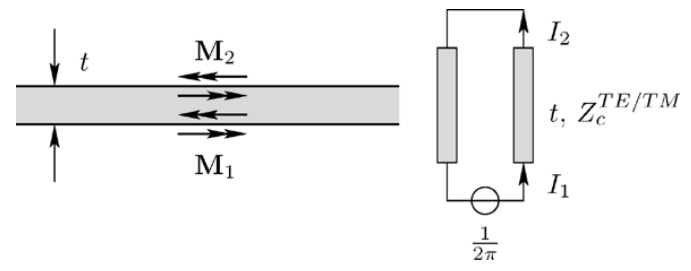

Fig. 2. Equivalent TL network for a parallel plate problem.

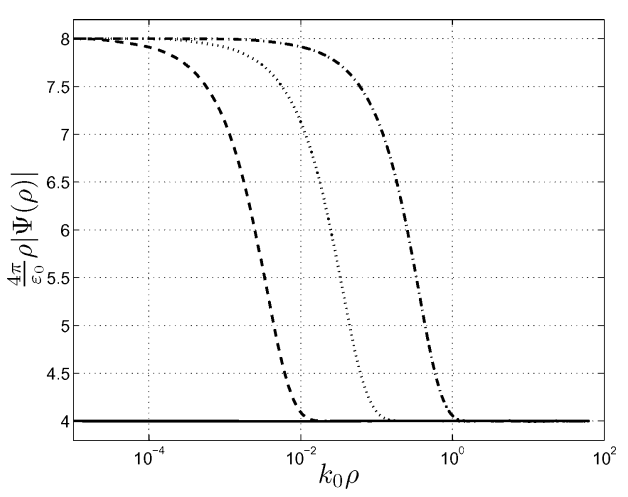

Fig. 3. Influence of the correcting term on the potential Green's function. Zero-thickness case (solid line), $t=\lambda / 1000$ (dashed line), $t=\lambda / 100$ (dotted line), $t=\lambda / 10$ (dash-dotted line).

verges to zero when $t \rightarrow 0$, ensuring this way a smooth transition to the zero thickness case.

The same approach remains valid for the multilayered stratified media inside the aperture. In this case the equivalent circuit will consist of a cascade of transmission lines with different characteristic impedances corresponding to different layers in the aperture.

Consider an aperture in a thick conducting screen separating two semi-infinite free spaces. Using the MPIE form, the IE will have a Green's function kernel that consists of

$$
\overleftrightarrow{\mathbf{G}}_{F}=\overleftrightarrow{\mathbf{G}}_{F}^{(1)}+\overleftrightarrow{\mathbf{G}}_{F}^{(2)}+2 \overleftrightarrow{\mathbf{G}}_{F}^{(c) \Delta}
$$

for the electric vector potential and

$$
G_{W}=G_{W}^{(1)}+G_{W}^{(2)}+2 G_{W}^{(c) \Delta}
$$

for the magnetic scalar potential. Fig. 3 depicts the normalized electric vector potential Green's function kernel $\Psi=G_{F}^{x x}=$ $G_{F}^{y y}$ computed using the presented approach for different aperture thicknesses, as a function of normalized distance.

\section{REsults FOR Thick SLOT CONFIGURATIONS}

Extensive numerical experiments have been carried out to assess the performance and limitations of the presented method. In this section we present comparisons against results obtained with a full-wave cavity approach and with measurements.

\section{A. Currents for a Plane Wave Excitation}

Let us consider a square aperture of dimensions $3 \lambda / 2 \times 3 \lambda / 2$ milled in a metallic plate of finite thickness. The aperture is assumed to be located in the air in the $x 0 y$ plane and to be illuminated by a plane wave impinging with the incident angle $\theta_{i}=0$ 


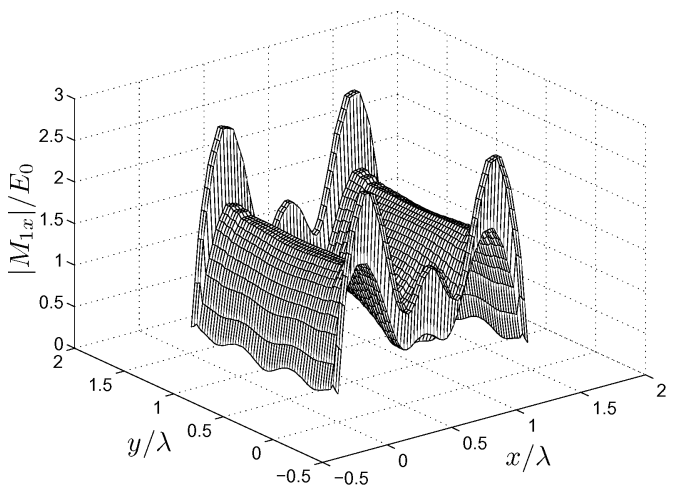

(a)

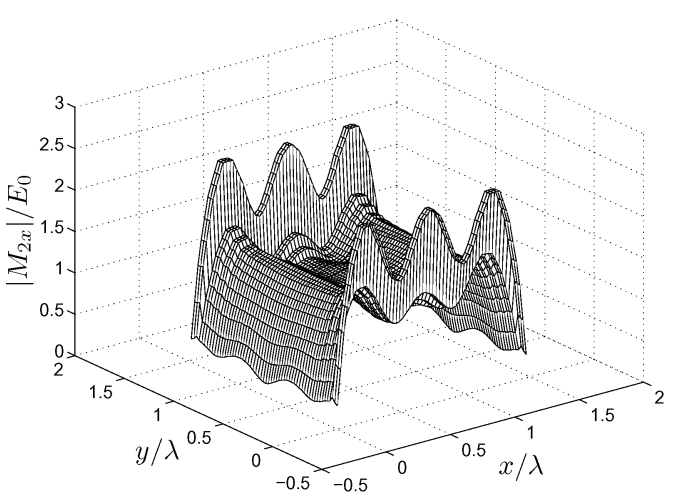

(b)

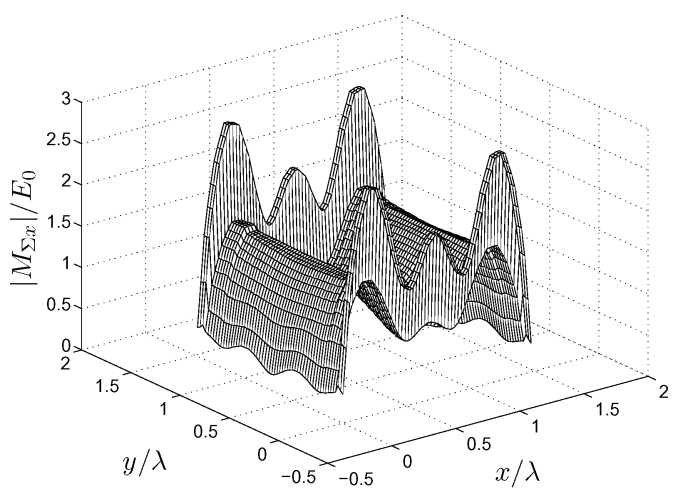

(c)

Fig. 4. Normalized magnitude of $x$-component of magnetic currents over $3 \lambda / 2 \times 3 \lambda / 2$ aperture of thickness $\lambda / 10$ illuminated by a plane wave impinging from below and having magnetic field polarized in $x$-direction. (a) Lower aperture interface (full cavity approach). (b) Upper aperture interface (full cavity approach). (c) Average value using this technique.

from below having the magnetic field polarized in the $x$-direction: $\mathbf{H}^{i}=\hat{x} H_{0} \mathrm{e}^{-j k z}$. In Fig. 4 the magnitudes of magnetic currents over the aperture are shown for the case of $\lambda / 10$ thickness computed using the full-wave cavity approach [7] and the presented approach. As can be seen, the shape of the magnetic currents on the top and bottom aperture interfaces is the same as of the magnetic current obtained with the presented approach. The $x$-components of magnetic currents over the lines $y=3 \lambda / 4$ (middle line) and $y=3 \lambda / 20$ (line close to the aperture border) are given in Fig. 5. The real parts of currents are shown with solid lines and the imaginary parts using dashed lines. $\mathbf{M}_{\Sigma}$ computed using our proposed approach is shown with diamonds,

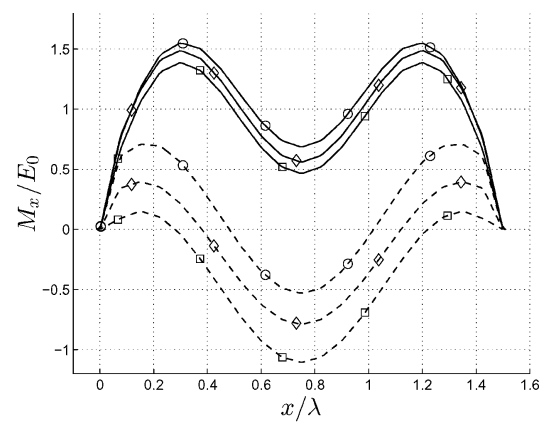

(a)

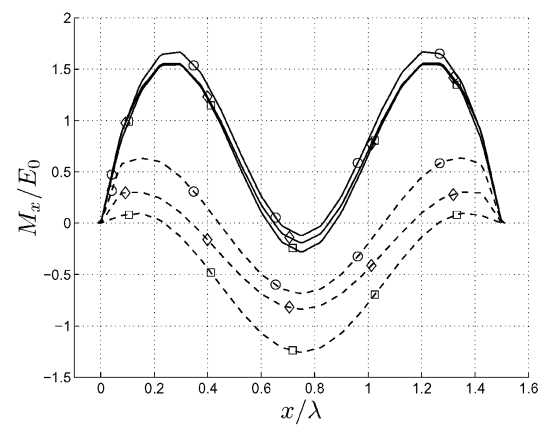

(b)

Fig. 5. Normalized magnetic currents over the square $3 \lambda / 2 \times 3 \lambda / 2$ aperture of $\lambda / 10$ thickness. ( $\diamond) \mathbf{M}_{\Sigma} ;(0) \mathbf{M}_{1} ;(\square) \mathbf{M}_{2}$. (Solid line) real part; (dashed line) imaginary part. (a) $y=3 \lambda / 4$ and (b) $y=3 \lambda / 20$.

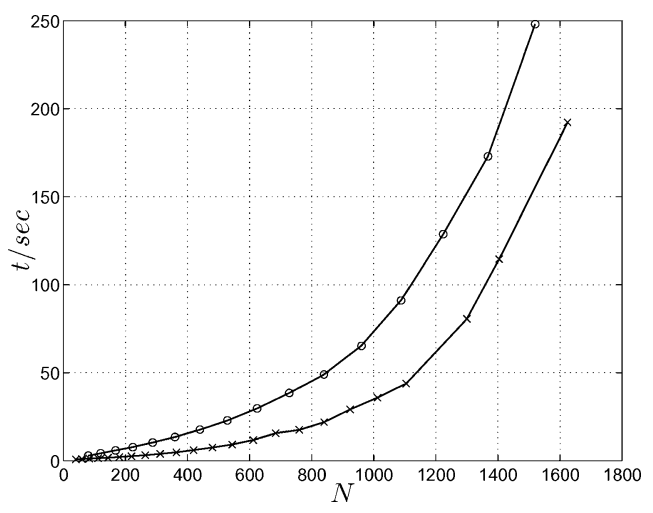

Fig. 6. Computational time versus number of unknowns for the presented approach $(x)$ and the full-wave cavity approach $(0)$ for $3 \lambda / 2 \times 3 \lambda / 2$ aperture of the thickness $\lambda / 10$.

while $\mathbf{M}_{1}$ and $\mathbf{M}_{2}$, computed using the full-wave cavity approach, are represented with circles and squares, respectively. The figure shows that both real and imaginary parts are close to half the sum of the currents on the lower and the upper interface of the aperture [Fig. 5(a)]. This relation is, however, deteriorated close to the aperture border [Fig. 5(b)].

In Fig. 6 the simulation time needed for solving the considered problem as a function of the number of unknowns is shown for both full-wave cavity approach (circles) and the approximate approach (crosses). Using the approximate approach, we have discretized the aperture in $20 \times 20$ cells, which corresponds to $N_{1}=760$ unknowns, and time needed for solving the problem on Pentium IV processor with $2.4 \mathrm{GHz}$ and $512 \mathrm{MB}$ of RAM was $t_{1}=17.6 \mathrm{~s}$. If we want the same discretization density, 


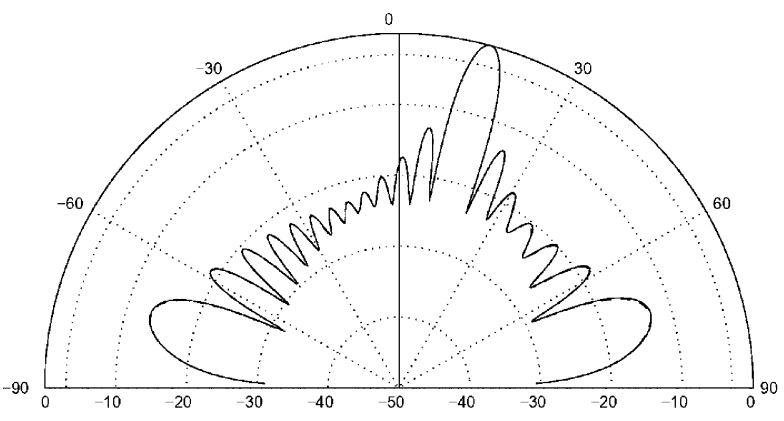

(a)

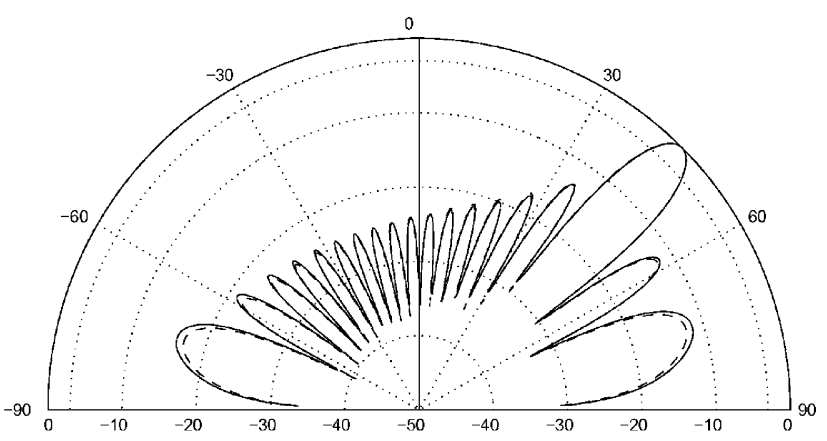

(b)

Fig. 7. Radiation patterns produced by currents induced by a plane wave impinging on the aperture from below for different thicknesses, computed using presented approach (solid lines) and full-wave cavity approach (dashed lines). (a) $t=\lambda / 100$ and incidence angle $\theta=15^{\circ}$. (b) $t=\lambda / 10$ and incidence angle $\theta=45^{\circ}$.

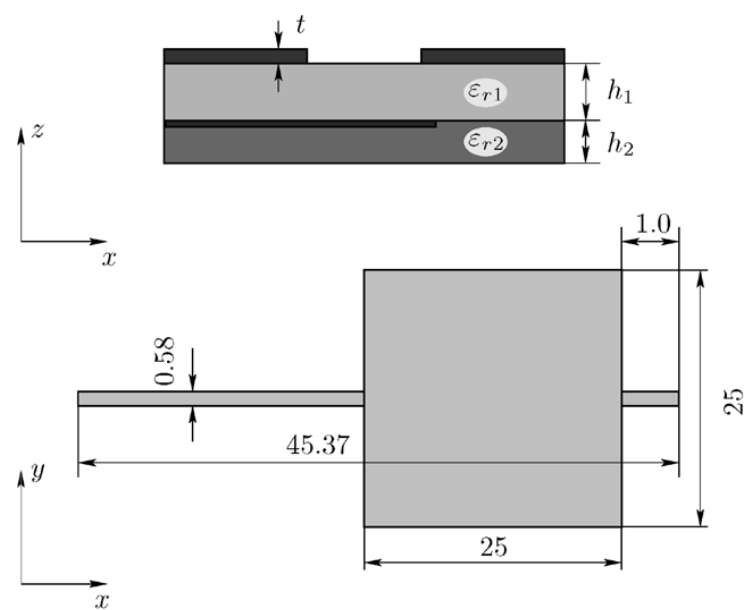

Fig. 8. Square thick aperture antenna. Dielectrics: $h_{1}=1.0 \mathrm{~mm}, \varepsilon_{r 1}=1.0$, $h_{2}=0.635 \mathrm{~mm}, \varepsilon_{r 2}=10.7, \tan \delta=0.0024$. All dimensions given in $\mathrm{mm}$.

using the full-wave cavity approach, we have to discretize both (upper and lower) aperture interfaces into $20 \times 20$ cells, which leads to twice as much the unknowns $N_{2}=2 N_{1}=1520$, and the time needed for solving the problem is $t_{2}=248 \mathrm{~s}$. The gain in computation time of 14 times is more than advantageous and is due to, on the one hand, the adoption of the parallel plate Green's function for the cavity which accounts for the bulk of the computational savings (as it obviates the time-consuming computation of the cavity Green's function). On the other hand, the number of unknowns is twice as small as we consider the aperture having only one interface.

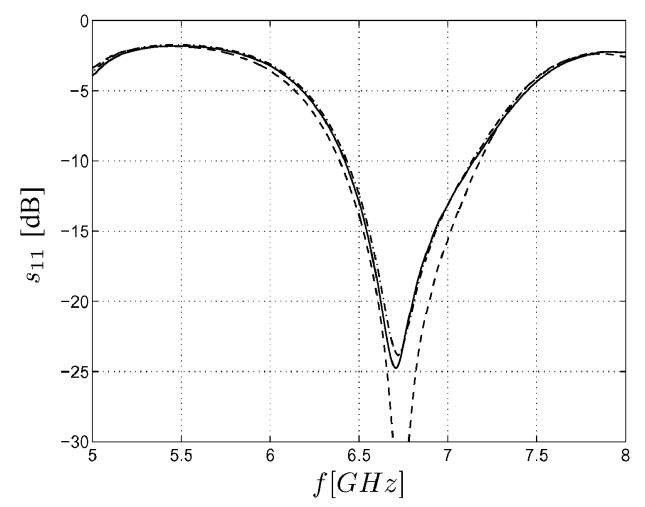

(a)

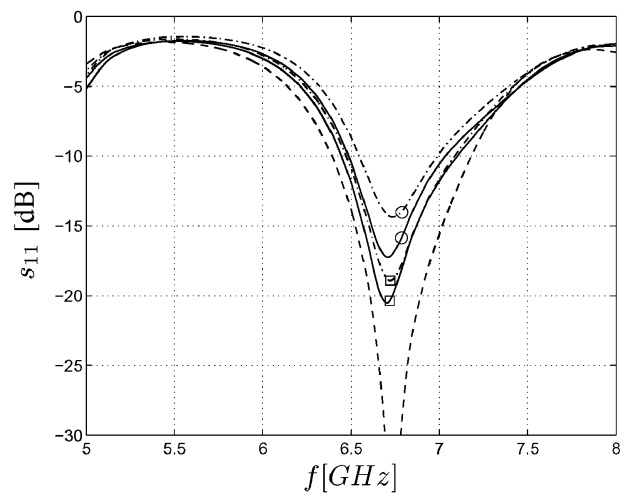

(b)

Fig. 9. Presented approach (solid line), full-wave cavity approach (dash-dotted line), and zero thickness case (dashed line). (a) $t=0.5 \mathrm{~mm}$. (b) $t=1 \mathrm{~mm}$ ( $\square$ ) and $t=2 \mathrm{~mm}(\mathrm{o})$.

\section{B. Scattered Fields for a Plane Wave Excitation}

In order to see the influence on the radiation pattern, the scattering on a rectangular slit of length $10 \lambda$ and width $\lambda / 10$, oriented with its longer side in the $x$-direction, has been simulated. Radiation patterns in the E-plane $\left(\phi=0^{\circ}\right)$ obtained using the presented and the full-wave cavity approaches are shown in Fig. 7. Two different incidence angles $\theta_{i}=15^{\circ}$ and $\theta_{i}=45^{\circ}$ of a plane wave with magnetic field polarized in the $x$-direction impinging from below were simulated. As expected, the difference between the two approaches in case of $\lambda / 100$ thickness can hardly be seen. In the case of $\lambda / 10$ thickness, the difference, although not excessive, is noticeable in the side-lobe levels.

\section{Comparison With a Full Cavity Rigorous Approach}

To test the accuracy and validity of the proposed approximate model, we have compared it with a rigorous full-wave cavity approach in the case of a square aperture electromagnetically fed by a microstrip line in a two-layer configuration (Fig. 8). In this case the incident field is modeled in a standard way using the delta-gap model and introducing half-rooftops along the edge the port is connected to. Fig. 9(a) gives the reflection coefficients obtained for a slot thickness of $0.5 \mathrm{~mm}(\sim 0.01 \lambda$ at the resonant frequency). For the sake of completeness, the results obtained by the standard zero thickness IE are also included. It can be seen that our model matches the full cavity model and hence predicts correctly the deviation from the zero thickness. 

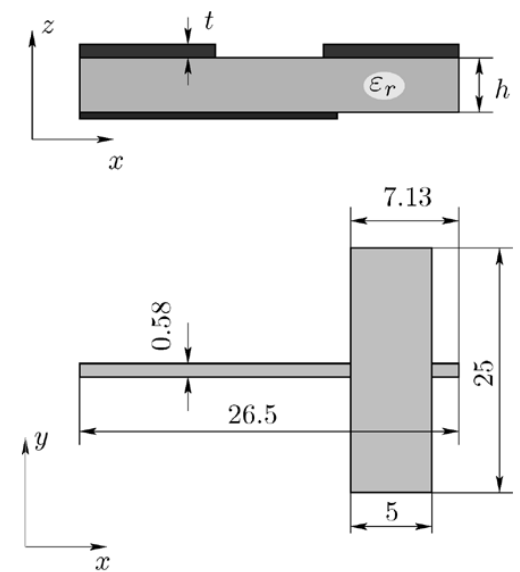

(a)

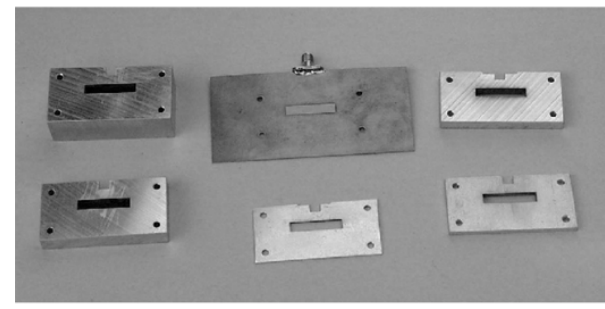

(b)

Fig. 10. Test antenna sample. A set of conducting screens of various thicknesses and with rectangular apertures can be fixed on the basic antenna. Dielectric made of RT-Duroid 6010: $h=0.635 \mathrm{~mm}, \varepsilon_{r}=10.5$, $\tan \delta=0.0024$. All dimensions given in $\mathrm{mm}$.

Fig. 9(b) presents similar predictions when the slot thickness is two and four times larger ( 1 and $2 \mathrm{~mm}$ ). As could be expected, here the predictions of the presented model start to deviate from those more rigorously obtained with the full cavity model, but the agreement is still reasonable.

\section{Thick Rectangular Aperture Antenna: Comparison With Measurements}

In order to check the results of the presented method against real-life measurements of input impedances, a set of rectangular aperture antennas with different aperture thicknesses has been realized (Fig. 10). The metallic screens are made of aluminum and the apertures are carefully manufactured by electric erosion, what guarantees very sharp corners of the rectangular shape. The aluminum bodies are mounted on the metallization of the substrate and they are tied together with plastic bolts. For the sake of reduced weight, the aluminum bodies do not cover the whole metallization of the substrate.

The computed and measured antenna input characteristics for two different metallization thicknesses $(t=1,3 \mathrm{~mm})$ are shown in Fig. 11. The substrate thickness (and hence the distance between electric currents in the feeding line and magnetic currents in the slot) is only $0.635 \mathrm{~mm}$ and is smaller than the thinnest slot considered $(t=1 \mathrm{~mm})$. Therefore, this is a very difficult case for our approach (where the effect of lateral electric walls in the slot is only included indirectly) and must be considered as a worst case revealing the limitations of our approach. However, the results presented in the Smith charts of Fig. 11 are still quite satisfactory, although the presented approach does not follow the measurements as well as the cavity approach,

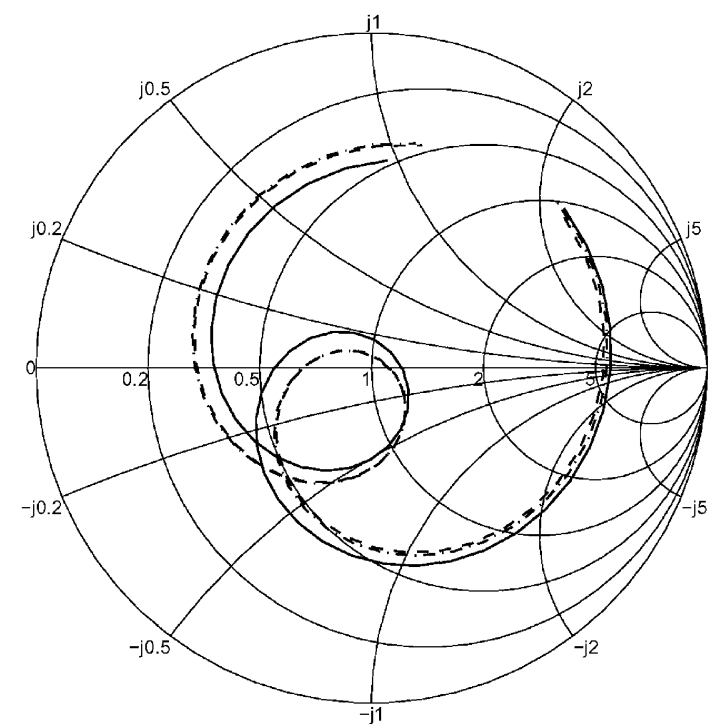

(a)

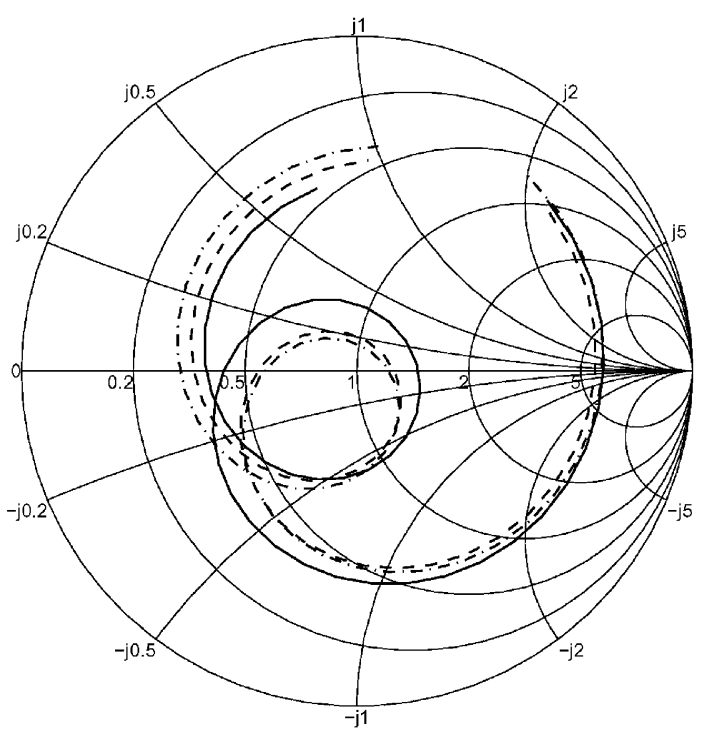

(b)

Fig. 11. Reflection coefficient of the rectangular aperture antenna. Measurements (dashed), presented approach (solid), and full-wave cavity computation (dash-dotted line). $f=5 \ldots 8 \mathrm{GHz}$. (a) Aperture thickness $t=0.02 \lambda=1 \mathrm{~mm}$. (b) $t=0.06 \lambda=3 \mathrm{~mm}$.

which is excellent in this situation, but at the expense of much more demanding computation time. The evaluation of the cavity Green's function is a very time-consuming process and results in about 10 to 20 times longer simulations, when compared to the code with the new formulation. So even in this worst case situation, the delta function remains an interesting alternative, and its predictions should improve dramatically when substrate layers thicker than the slots are used, as predicted in the previous section.

\section{E. Thick Dog-Bone Aperture Antenna: Comparison With Measurements}

A more general shape of the thick aperture antenna is investigated. The layout of the antenna is shown in Fig. 12(a). The aperture is now extended to a dog-bone-type shape, which can 

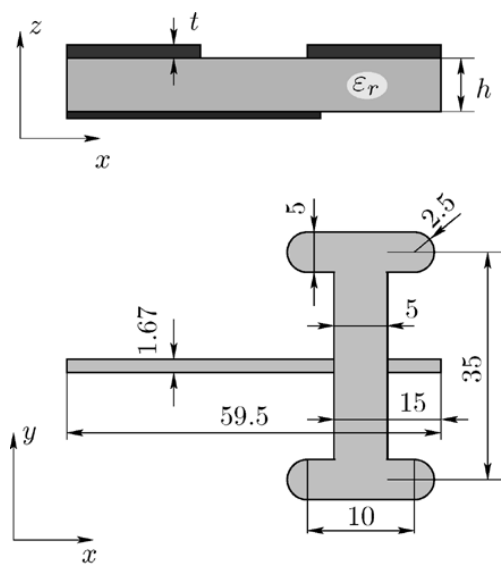

(a)

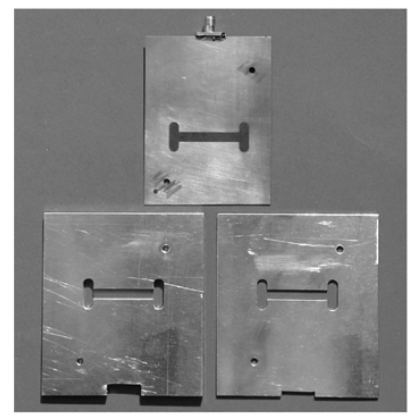

(b)

Fig. 12. Dog-bone aperture antenna. Dielectric: Duroid5870, $\varepsilon_{r}=2.33$, $\tan \delta=0.0012, h=0.51 \mathrm{~mm}$. All dimensions given in $\mathrm{mm}$. (a) Layout of the antenna design. (b) Picture of the realized antenna samples.

be easily milled in a metallic shield. Fig. 12(b) shows the realized antenna with two thick metallic shields of $t=1$ and $3 \mathrm{~mm}$.

The results of the three samples, with thicknesses $t=0,1,3$ $\mathrm{mm}$ are shown in the Smith charts of Fig. 13. Here it must be pointed out that already for zero thickness slot [Fig. 13(a)], the theoretical prediction does not follow as closely the measurements as in the case of rectangular slot antenna. This shows clearly the numerical degradation associated with the modeling a complicated shape like the dog-bone. The good news is that, despite the thin substrate $(h=0.51 \mathrm{~mm})$, the presented approach does not seem to degrade the performances of our IE code and the agreement remains quite fair. In particular, the qualitative trend of the input impedance resonant loop opening with increased aperture thicknesses is perfectly predicted.

\section{CONCLUSION}

In this paper, we have presented the numerical and experimental study of an approximate technique for numerical treatment of scattering from apertures in metal screens with finite thickness [11]. The approach reduces significantly the simulation time needed when a full-wave cavity treatment of the thick apertures is employed. Various tests with increasing slot thicknesses have been performed and studied. In conclusion, it can be said that the new method yields accurate prediction on the input impedance up to metallization thicknesses of about $t=0.1 \lambda$. A point that deserves further research is the source of these limitations. The original set of coupled integral equations

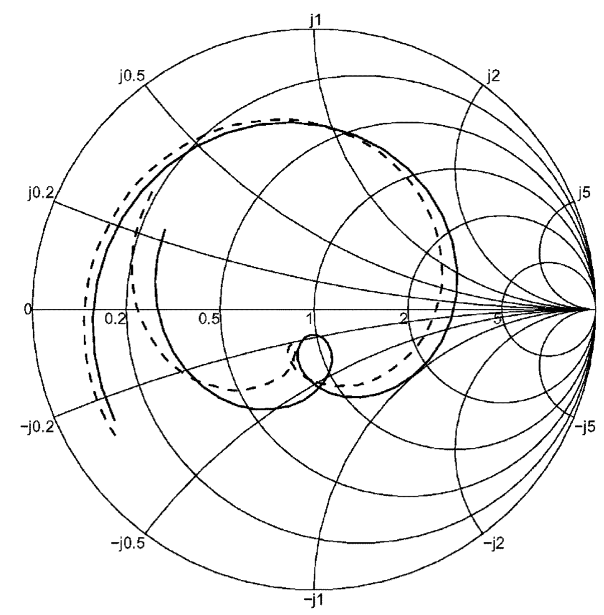

(a)

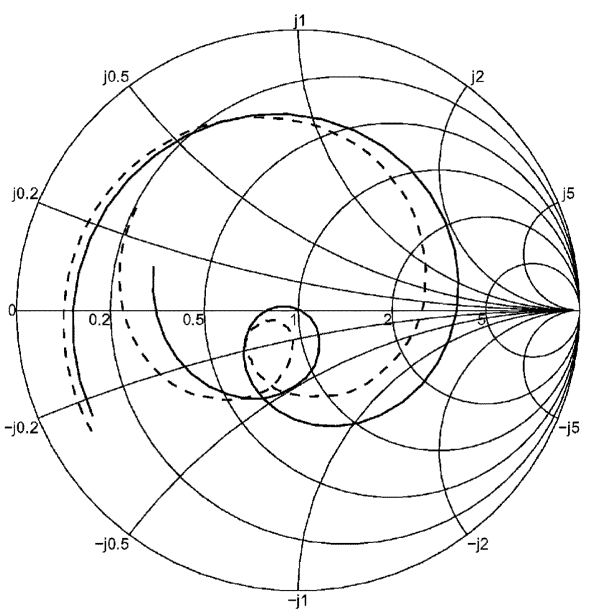

(b)

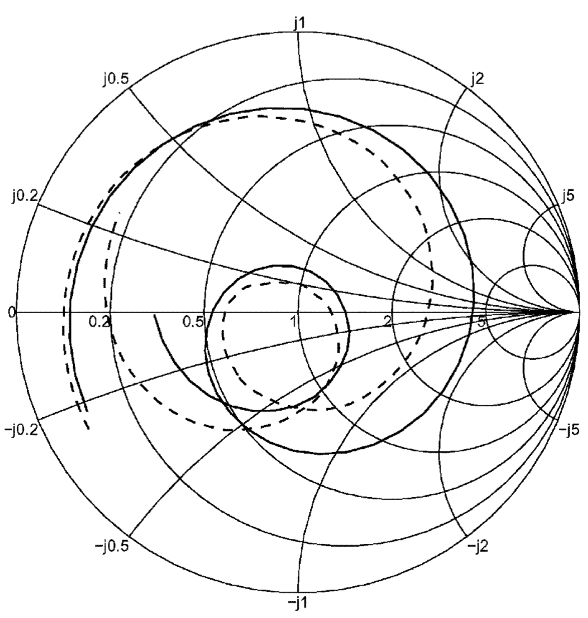

(c)

Fig. 13. Dog-bone aperture antenna, $f=3 \ldots 6 \mathrm{GHz}$. Measurements (dashed line) versus simulation (solid line) for various aperture thicknesses. (a) Thickness $t \approx 0 \mathrm{~mm}$. (b) $t=1 \mathrm{~mm}=0.015 \lambda_{0}$. (c) $t=3 \mathrm{~mm}=0.045 \lambda_{0}$.

presented in this paper is rigorous and exact from a full-wave point of view. There are two approximations introduced. One approximation has been introduced by replacing the cavity by parallel-plate Green's functions. However, this could not be the real limiting factor for the presented approach, but rather 
the fact that the difference magnetic current $\mathbf{M}_{\Delta}$ has been also neglected. Therefore, solving iteratively the set of coupled equations starting with $\mathbf{M}_{\Delta}=0$ as initial situation could even enlarge the validity and scope of the presented method. Results will be reported in a coming paper. In any case, it must be pointed out that the upper limit reached in the current status of the method is already highly satisfactory. Many technologies using self-supporting metallic patches in $K a$ and $K u$ bands or thick conducting film in millimeter and submillimeter wave bands yield electrical thicknesses within this limit.

\section{REFERENCES}

[1] R. Harrington and J. Mautz, "A generalized network formulation for aperture problems," IEEE Trans. Antennas Propagat., vol. AP-24, pp. 870-873, Nov. 1976.

[2] M. Himdi, J. Daniel, and C. Terret, "Analysis of aperture-coupled microstrip antenna using cavity method," Electron. Lett., vol. 25, pp. 391-392, Mar. 1989.

[3] O. Lafond, M. Himidi, and J. Daniel, "Aperture coupled microstrip patch antenna with thick ground plane in millimeter waves," Electron. Lett., vol. 35, pp. 1394-1395, Aug. 1999.

[4] P. Haddad and D. Pozar, "Characterization of aperture coupled microstrip patch antenna with thick ground plane," Electron. Lett., vol. 30, pp. 1106-1107, July 1994.

[5] J. Jin and J. Volakis, "Tm scattering by an inhomogenously filled aperture in a thick conducting plane," Proc. Inst. Elect. Eng., vol. 137, pp. 153-159, June 1990.

[6] S. Gedney and R. Mittra, "Electromagnetic transmission through inhomogenously filled slots in a thick conducting plane-arbitrary incidence," IEEE Trans. Electromagn. Compat., vol. 34, pp. 404-415, Nov. 1992.

[7] A. Alvarez-Melcon and J. R. Mosig, "An efficient technique for the rigorous analysis of shielded circuits and antennas of arbitrary shapes," in JINA'98 Int. Symp. Antennas, Nov. 17-19, 1998, pp. 57-60.

[8] —, "Two techniques for the efficient numerical calculation of the green's function for planar shielded circuits and antennas," IEEE Trans. Microwave Theory Tech., vol. 48, pp. 1492-1504, Sept. 2000.

[9] J. R. Mosig and A. Alvarez-Melcon, "The summation-by-parts algorithm - A new efficient technique for the rapid calculation of certain series arising in shielded planar structures," IEEE Trans. Microwave Theory Tech., vol. 50, pp. 215-218, Jan. 2002.

[10] D. L. del Río and J. R. Mosig, "On the interaction of magnetic and electric currents in stratified media," IEEE Trans. Antennas Propagat., vol. 52, pp. 2100-2108, Aug. 2004.

[11] J. R. Mosig, "Scattering by arbitrarily-shaped slots on thick metallic screens: An approximate solution," IEEE Trans. Antennas Propagat., vol. 52, pp. 2109-2117, Aug. 2004.

[12] C. M. Butler, Y. Rahmat-Samii, and R. Mittra, "Electromagnetic penetration through apertures in conducting surfaces," IEEE Trans. Antennas Propagat., vol. AP-26, pp. 82-93, Jan. 1978.
[13] J. V. Bladel, Electromagnetic Fields. New York: McGraw-Hill, 1964.

[14] R. Collin, Field Theory of Guided Waves, 2nd ed. New York: McGrawHill, 1991.

[15] K. Michalski and J. R. Mosig, "Multilayered media green's functions in integral equation formulations," IEEE Trans. Antennas Propagat., vol. 45, pp. 508-519, Mar. 1997.

[16] J. R. Mosig, "Integral-Equation Technique," in Numerical Techniques for Microwave and Millimeter-Wave Passive Structures. New York: Wiley Interscience Publication, 1989, ch. 3, pp. 133-213.

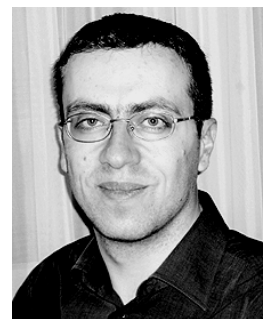

Ivica Stevanović (S'03) was born in 1976 in Kruševac, Yugoslavia. He received the Dipl.Ing. degree from the School of Electrical Engineering (ETF), Department of Electronics, Communications and Control, University of Belgrade, in 2000 . He is currently pursuing the $\mathrm{Ph} . \mathrm{D}$. degree at the Laboratory of Electromagnetics and Acoustics (LEMA), Ecole Polytechnique Fédérale de Lausanne (EPFL), Switzerland.

He was a Student Assistant at the Laboratory of Electronics of ETF (1998-2000). In November 2000 he joined LEMA, EPFL, where he is currently a Research and Teaching Assistant.

Mr. Stevanović received a scholarship from the Serbian Ministry of Education (1996-2000) and a research fellowship (SURF) from the California Institute of Technology, Pasadena (summer 2000).

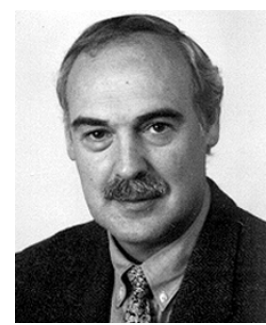

Juan R. Mosig (S'76-M'87-SM'94-F'99) was born in Cadiz, Spain. He received the electrical engineer degree from Universidad Politecnica de Madrid, Spain, in 1973 and the Ph.D. degree from the Laboratory of Electromagnetics and Acoustics, Ecole Polytechnique Fédérale de Lausanne (EPFL), Switzerland, in 1983.

Since 1991, he has been a Professor at EPFL. In 1984, he was a Visiting Research Associate at Rochester Institute of Technology, Rochester, NY. $\mathrm{He}$ has also held scientific appointments at universities of Rennes (France), Nice (France), Technical University of Denmark, and University of Colorado, Boulder. He is the author of four chapters in books on microstrip antennas and circuits. He is a Coorganizer and Lecturer of yearly short intensive courses in numerical electromagnetics (Europe and United States). His research interests include electromagnetic theory, numerical methods, and microstrip antennas. He is responsible for several research projects for the European Space Agency.

Prof. Mosig is a Member of the Swiss Federal Commission for Space Applications. 